\title{
On the Factors Influencing L1 Transfer
}

\author{
Aisong Yi \\ School of Foreign languages, Jiangsu University, Zhenjiang 212013, China \\ Email: yiaisong@ujs.edu.cn
}

\begin{abstract}
For decades, the phenomenon of language transfer has been a focus of second language acquisition. The study of language transfer has experienced three stages. It has long been noted that the linguistic differences between $L 1$ and $L 2$ will affect the acquisition of $L 2$, both positively and negatively. This paper aims at sorting out factors that are facilitating to $\mathrm{L} 1$ transfer. Seven categories of factors have been examined: Linguistic factors, Psycholinguistic factors, Sociolinguistic factors, Socio-psychological factors, Individual difference, Developmental factors, and Frequency of input. Giving the complexity of transfer phenomenon, though the above-mentioned factors are discussed separately, the need to investigate their co-influence on L1 transfer is suggested.
\end{abstract}

Index Terms - L1 transfer, factors, facilitating

\section{INTRODUCTION: HiSTORIC REVIEW AND CURRENT UNDERSTANDING OF LANGUAGE TRANSFER}

For decades, the phenomenon of language transfer has been a focus of second language acquisition. Historically, the study of language transfer has experienced three stages: the first stage is from 1950s to 1960s, when the study of language transfer was strongly influenced by behaviorism. At that time, the strong version of contrastive analysis hypothesis (CAH) asserted that through careful comparison of the native language (NL) and target language (TL), the difficulties in TL acquisition could be predicted. The strong version of CAH was proved to be unable to fulfill what it had claimed to do, however; thus, with the growing disfavor of behaviorism, CAH received strong criticisms, especially from mentalists during the late 1960s. And from then on, the study of language transfer entered into the second stage up to 1970s. During that period, under the influence of Chomsky's UG (universal grammar) theory, and with the revolutionary studies conducted by Dulay and Burt, the role of language transfer in the process of L2 (second language) acquisition was considered to be trivial. The third period of language transfer study, from 1980s up to date, is marked by the introduction of multidisciplinary perspectives into the field of SLA (second language acquisition) research; consequently our understanding toward the language transfer phenomenon has been deepened.

Now, as pointed out by Selinker (1992), the consensus view of language transfer is that it is not an "all or nothing" phenomenon. Actually, transfer has been regarded as a very broad concept of cross-linguistic influence not confined within the scope of L1 and L2. Odlin (1989) has proposed a working definition of transfer: Transfer is the influence resulting from similarities and differences between the target language and any other language that has been previously (and perhaps imperfectly) acquired.

Similarly, Selinker (1992) has concluded that: Language transfer is best thought of as a cover term for a whole class of behaviors, processes and constraints, each of which has to do with CLI (cross linguistic influence), the influence and use of prior linguistic knowledge, usually but not exclusively NL (native language) knowledge. This knowledge intersects with input from the TL (target language) and with universal properties of various sorts in a selective way to help build IL (interlanguage).

Such an understanding of language transfer presupposes that the analysis language transfer needs to be approached from different perspectives.

\section{What Results IN L1 TRANSFER?}

\section{A. Linguistic Factors}

\section{Language distance}

It has long been noted that the linguistic difference between L1 and L2 will bring difficulties in the acquisition of L2. Lado (1957) has proposed the CAH (contrastive analysis hypothesis) in which the predicted positive transfer and negative transfer in L2 learning process are solely based on the structural relationship between the languages in comparison. Yet numerous facts indicating that the difficulties predicted by the $\mathrm{CAH}$ do not prove to be difficulties at all has aroused strong criticism on CAH. Today, we have formed an objective view toward the difference between two languages, as Ellis (1994) has noted, 'language distance can affect L2 learning both positively and negatively'. (p.338) Scholars have detected the different manifestations of the result of L1 transfer, with some of them are conspicuous and some of them hard to be identified. For instance, Ringbom (1978) and Sjoholm (1976) have concluded that the acquisition of lexis appears to be facilitated if the L1 and L2 are related languages. Another example came form Schachter (1974), who has found that Chinese and Japanese learners of L2 English made fewer errors in their use of 
relative clauses than Persian or Arabic learners because they produced far fewer clauses overall-L1 transfer is manifested in terms of (communication or learning) strategies adopted by L2 learners. All these evidence indicate that a native-target language comparison is nonetheless an important preliminary step to understand language transfer.

2. Language universal: markedness

Ellis (1994) suggests that the transferability of different features depends on their degree of markedness. Markedness, according to Ellis, refers to the idea that some linguistic structures are "special" or "less natural" or "less basic" than others. There are basically two approaches to the definition of markedness. One is derived from Chomsky's UG (universal theory), the other is originated from the study of typology initiated by Greenberg.

Chomsky distinguishes core rules of a language from that are peripheral. According to him, core rules are those that can be arrived at through the application of general, abstract principles of language structures, which he believes to be innate; peripheral rules are those not governed by universal principles, they are unique in a specific language. While the core rules are unmarked, the peripheral rules are marked.

Empirical studies of L2 acquisition based on the definition of markedness within the UG framework have generated divergent results. Ellis attributes such separating results to the lack of consensus about the details of the theory, and suggests that it is premature to reach any conclusion as to whether markedness, as defined by the theory of UG, is a relevant factor in L2 acquisition.

Another definition of markedness, which has been widely used by scholars to explain L1 transfer phenomenon, comes from the study of language typology. According to Ellis (1994) the broad claim of the definition is that those features that are universal to present in most language are unmarked, while those that are specific to a particular language or found only in a few languages are marked. Such an understanding of markedness indicates that markedness is better to be understood as a relative concept-there may be few absolute universals (universals that are exemplified in all languages), but universal tendencies may be more common.

The markedness theory is certainly useful for our understanding of L1 transfer phenomenon. Yet it is not an omnipotent theory free of deficiency. One of the problems of the theory, as pointed out by Ellis is the vagueness of the concept which sometimes makes it difficult to determine which features are marked in relation to others. Ellis goes on to suggest that the concept could be more precise if it is defined with reference to 'native speakers' own perception of the structure'. In deed, as indicated by Kasper and Faerch (1987), with the purpose of reconstructing transfer procedures as they operate in learners' minds, psychological and social-psychological dimensions should be taken into consideration. The following two sections are devoted to this regard.

\section{B. Psycholinguistic Factors}

While commenting on CAH, Long and Sato (1984) have pointed out that the scholars of CAH have attempted to yield meaningful insight into a psycholinguistic process, i.e. L2 learning based solely on an analysis of linguistic product.

Discussing the deficiency of the CAH is not the focus of this paper, however; yet the comment indicates that the language transfer is more of a psychological problem than of a linguistic one. As Ellis has suggested, current definitions of the term "transfer" allow psycholinguistic L1 effects, thus any discussion of L1 transfer without addressing the psychological aspect if it is incomplete. Here we will focus on the two psycholinguistic factors: Prototypicality and Psychotypology

Kellerman is among the first to address the psychological aspect of L1 transfer phenomenon. In a series of studies, he demonstrated that native speakers' intuition about semantic space can be used to predict transferability. He proposed a term "prototypicality" to refer to the perceptions that learners have regarding the structure of their own languages: A feature is marked if it is perceived as infrequent, irregular, semantically or structurally opaque, or in any other ways exceptional. Such perceptions will in turn lead them to treat some structures as transferable and others as non-transferable. Based on this, a hierarchy of psychological "markedness" is possible. Actually, as indicated by Kasper and Faerch (1987), the degree of markedness of an L1 feature is an important factor in determining whether this feature is considered transferable.

Though the conclusion of Kellerman seems to be persuasive, the weakness of this approach is obvious, as pointed out by Ellis (1994: 327), following this approach, "we do not know to what extent learners' judgments about what can be done accurately reflect what they actually do when using the L2". That is to say, "translatability" does not necessarily equal to "transferability".

Later, Kellerman (1978) proposed the concept of psychotypology, claiming that learners' perception of the distance between their native language and the target language could be a crucial factor in determining whether they transfer or not. Kellerman (1979) argues that learners' psychotypology is not fixed; rather, it is revised as they obtain more information about the target language.

\section{Sociolinguistic Factors}

In so far we have discussed linguistic and psycholinguistic factor that are conducive or prohibitive to the phenomenon of language transfer, a discussion could never be sufficient so long as the fact that transfer manifests in communicative interaction, is ignored.

It has been argued that IL is characterized by viability, and that such viability is systematic, corresponding to 
contextually determined variability in the native language. If that is true, then the question followed would be how different IL varieties are activated in different contexts. Tarone suggests that L2 learners' performance constitutes a continuum in accordance with different contexts, with the "vernacular" at one head of the continuum and the "careful" style at the other. Her study, along with that of Dickerson's, indicate that learners' performance tend converge to TL norms when the "careful" style is adopted and their performance tend to deviate from TL norms when the "vernacular" style is adopted.

Researches conducted by Odlin (1989) and Tarone (1982) suggest that L1 transfer is connected with different contexts, thus relating to different IL varieties; however, their conclusions seems to be incompatible with each other as to suggest under what contexts, to which extent, transfer would occur. Odlin (1989) has argued that native transfer is less likely in focused contexts, where there is concern to maintain the standardness of languages, than in unfocused contexts. For example, he suggests that negative transfer is more likely to occur off class than on class. While he approaches the sociolinguistic factor on transfer from a macro-sociolinguistic perspective, i.e. how external environments exert different demands on language learners in terms of "standardness", Tarone (1982) takes a micro-sociolinguistic perspective, i.e. how learners adapt their performance to fulfill different communication tasks in accordance with different external environments. She argues that L1 transfer is likely to be more evident in learners' careful style than in their vernacular style, because they are more likely to make use of all their potential resources, including L1 knowledge.

Obviously, Odlin's position is in contradiction to that of Tarone's. Ellis has pointed out the danger of discussing the influence that sociolinguistic factors have on language transfer in terms of solely micro or solely macro perspective. Instead, he suggests that it is necessary to take into account of both internal and external norms that learners need to conform in various contexts. While Ellis's comment is a pertinent one, it does not seem to be a very practical one - the relative weight perceived by individuals may differ from one person to another, and also from one context to another; further, it may also involves other factors such as social-psychological consideration (which will be discussed below), or personality orientation, thus making the analysis of L1 transfer fairly complex. Investigation to the interplay (of different factors) determining L1 transfer is thus in need.

\section{Social-psychological Factors}

We believe that the term social-psychological is different from the term social-linguistic, although the two are clearly related to each other. We believe social-psychological factors in many cases are value-laden; therefore they operate at a deeper level than do socio-linguistic factors.

The manifestations of the influences of socio-psychological factors on L2 speakers' behavior are strategic in nature. In this sense, it could be said that some of the socio-psychological factors are strategic factors, which incorporate both learning and communication aspects; yet clearly, from the discussion above, social-psychological factors are more than strategic factors.

Kasper and Faerch (1987) has proposed three social-psychological factors which lead L2 speakers not to produce as correct a variety of their IL as (cognitively and linguistically) possible in a given situation. In fact the social-psychological factors proposed by Kasper and Faerch is better to be understood as a enlarged and deepened discussion of what Tarone has labeled as micro-sociolinguistic perspective. The three factors are: group solidarity, foreigner role, and marking origin.

The first type of factor is group solidity; a strong sense of group solidity in L2 speech community would lead L2 speakers to retain in their IL features of their social / ethnic identity---group solidity result in divergent behavior because of a desire to distance oneself from one's interlocutor. The second type of factor is foreigner role. While the maintenance of group solidarity is meant to protect L2 speakers' own values from being questioned in the TL community, the assumption of the foreigner role is meant to protect themselves from being judged on the basis of native-speaker norms and expectations, and therefore creating a positive learning environment for L2 acquisition, and L2 communication as well.

The third type of factors is marking origin. As indicated by Kasper and Faerch, this is 'a rather special case of L1 transfer within a socio-psychological perspective', because it 'occurs when 'commodities' (in a broadest possible sense of the term) originating in one culture are being transposed to a different culture'.

It is interesting to note from the above discussion that L2 speakers influenced by socio-psychological factors tend to transfer in a conscious manner, while under the influence of other factors discussed before such as language universal and psychotypology, L2 speakers are inclined to transfer in a subconscious manner.

\section{E. Individual Difference in Terms of Personality}

The preceding discussion of L1 transfer is carried out at the collective level rather than at the individual level, that is, we have focused on the behavior of a group of, instead of single, L2 speakers. Many scholars have noticed individual difference in L2 acquisition, however. Odlin have reminded us of the fact that the manifestation of transfer can vary from one learner to the next, even if some kinds of transfer is likely in the second language performance of most learners. Thus, any discussion of L1 transfer will not be sufficient without giving any consideration to individual variation.

We find individual difference such a broad concept that a total account of it is beyond the scope of the paper. Relating 
to L1 transfer, we will focus on personality only. Odlin acknowledges that some, if not all, personality difference tend to increase or decrease the likelihood of transfer. He summarizes, based on studies of Schachter, Kleinmann, and Guiora (1972), that anxiety and empathy are two personality characteristics that seem to interact with transfer. As for anxiety, those who are more susceptible to anxiety tend to avoid unfamiliar structures of the TL, thus, they may resort more to their NL than their counterparts who are less susceptible to anxiety.

While the difference in the susceptibility of anxiety could explain whether individual L2 speakers would use a specific TL structure, the varied degree of empathy among L2 learners may account for the varying degrees of success that individuals have in approximating native-like proficiency. Guiora's (1972) study of the L2 pronunciation has suggested that 'individual differences in the ability to approximate native-like pronunciation should reflect individual differences in the flexibility of psychic processes, or more specifically, in the empathetic capacity'.

It is highly likely, following Kellerman's characterization, that if learners are more aware of their own cultures and linguistic norms, they would allow or retain more transfer from their native language to their IL than learners who have less awareness of their own cultural and linguistic norms, and vice versa. It is thus possible the less an individual learner can feel emotionally 'inside' the target language speech community, the more likely he/she would manifests L1 transfer.

\section{F. Developmental Factors}

Coder (1978) considers the acquisition of L2 as a restructuring process with L2 features gradually replaces that of L1. It follows that negative transfer is more evident at the beginning stage, and such a position is supported by the studies of Major (1986) and Wenk (1986) concerning L2 phonology acquisition. However, as Ellis (1994) has noted, not all errors in early interlanguage are traceable to transfer - many are intralingual and resemble those found in L1 acquisition. Also, some error traceable to L1 influence, only come out at later stages of development. Further, it is not necessary that transfer errors which appear at an early stage of development are subsequently eliminated.

Whereas some researchers have suggested that transfer is more associated with early stages of L2 acquisition, others have argued that learners may need to reach a certain stage of development before transfer of some L1 properties become possible. Ellis (1997) has found it clearly evident in the way learners acquire speech acts like requests, apologies, and refusals. Learners do not initially transfer their L1 speech-act strategies but, instead, rely on a few simple formulas. Later, however, as learners' L2 proficiency develops, they may try to find ways of performing speech acts that accord with L1 forms.

There is growing evidence suggesting that the L1 and developmental factor work together in determining the course of interlanguage or, as Zobl (1980) has noted, transfer is selective along the developmental axis. Ellis (1994) indicates the selectivity is evident in three ways

1. the effect of the L1 only become evident when the learner has reached a stage of development that makes transfer possible

2. development may be retarded when a universal transitional structure arising naturally in early interlanguage corresponds to an L1 structur

3. development may be accelerated when an early transitional structure is not reinforced by the corresponding L2 structure

It is clear that transfer interacts with natural principle of L2 acquisition, sometimes occurring early on and sometimes later, it can both retard and accelerate natural development.

\section{G. Frequency of Input}

Since the research conducted by Hatch and Wagner-Gough (1976), it has been noticed that the frequency of L2 input is related to its acquisition. Thus we have a so called frequency hypothesis which states that the order of L 2 acquisition is determined by the frequency with different linguistic items occur in the input. As noted by Ellis (1994) the frequency hypothesis is meant to deal with the relationship between input and accuracy, and the justification of the hypothesis is based on the assumption that the order of accuracy equals to that of acquisition.

While the underlying assumption of the frequency has been subjected to question on one hand, results of researches conducted under the theoretical framework have also indicated divergent results. As Larsen-Freeman and Long (1991) has noted 'there exist preliminary data supporting a frequency effect'. Ellis (1994) holds the similar view and further suggests that 'it is possible that frequency may be more important at some stages of acquisition (for example, elementary) than others, but no clear conclusion is possible on the basis of these (divergent) studies'. (p. 271)

It is not the purpose of this paper to discuss the deficiency of the frequency hypothesis, in the sense that the hypothesis is not meant to address the problem of L1 transfer. Yet another newly developed theory, which is fundamentally different from the frequency hypothesis, the PDP (Parallel Distributed Processing) model, or the connectionist theory, based on probabilistic patterns, has offered us a new perspective to understand the role of input frequency of in L2 learning.

Scholars of the connectionist theory posit that the brains of human beings are endowed with the inclination of searching and establishing connections between different things. The nerve fibers inside the brain are connected with each other to form a network; the connections between the nerve fibers will gradually be strengthened if it receives incessant activation, weakened if little activation is assigned to that connection. The learning process is the one in which the weight of the connections between the network is gradually altered; learning process is not the one that abstract 
rules are gradually established.

According to the connectionist theory, language learners will notice the regularities in the TL input, that is, they will notice that some elements or phenomena occur more frequently than others. After this, language learners will be able to abstract probabilistic patterns from TL input. The probabilistic patterns will gradually be strengthened for repeated activation; thereby language acquisition is made possible. With such an idea, it seems to be true that the so called rules or principles of a language are actually operating on the basis of probability. The more frequent a feature occurs in TL input, the easier it is to be acquired.

Following the connectionist theory, it seems to be safe to conclude that L1 constitutes one of the major difficulties of L2 acquisition. Further, L1 transfer could thus be viewed as a consequence of the activation of L1 probabilistic patterns triggered by $\mathrm{L} 2$ input.

\section{THE Role OF L1 IN L2 ACQUiSITION}

When discussing the role of L1 in L2 acquisition, we are interested in how L1 knowledge interacts with input in shaping the learner's IL system and how both L1 and IL knowledge are drawn on in L2 production. Ellis has pointed out the necessity of distinguishing two types of transfer, namely, communication transfer and learning transfer, as they represent different approaches in studying L1 transfer phenomenon.

Scholars such as Corder (1983) attempt to explain L1 transfer in terms of solely communication, they deny the idea that learners directly transfer into their interlanguage system L1 elements. Corder used the term 'borrowing' to label L1 transfer, indicating the later is mainly a communication strategy. He believes that 'nothing is being transferred from anywhere to anywhere'. However, such a view of transfer is problematic, as indicated by Ellis in the sense that particular transfer errors occur in whole populations sharing the same L1, it seems to be inappropriate to suggest that all these learners engaged persistently in borrowing and as a result learnt the L1 structure; further, it is also unclear how communication transfer can explain the fossilization of certain L1 influenced structures in learners' IL of the kind that Kellerman have identified in advanced Dutch learners of English .

Therefore, it is not apropos to study transfer from the communicative perspective only. The need to recognize a more direct role of L1 in L2 acquisition is proposed by Schachter, who offered 'a new account of transfer', suggesting the regularity of the occurrence of learning transfer, as well as the need to understand the leaning transfer from a cognitive perspective rather than from a behaviorist perspective. She regards transfer as the set of constraints that one's previous knowledge imposes on the domains from which to select hypotheses about the new data. She uses the term 'universe' to refer to 'the set of constraints' and suggested that a learner's universe is dynamic in nature, i.e. it expands or contracts with the accumulation of learner's experience with L2. Because learner's L1 knowledge is part of his 'previous knowledge', it thus constitutes a very important source of knowledge from which a learner could draw upon in forming (and testing) new hypotheses about L2 along its development course.

Thus, the role of L1 in L2 acquisition should be approached from the viewpoint of both communication and learning. Ellis has offered a framework for explaining first language transfer in second language learning and communication.

Within the model, Ellis has proposed the following points:

1. The L1 system is utilized by both comprehension and production mechanisms, in both cases, there are constraints that govern when transfer takes place.

2. The interlanguage system is also utilized in the process of comprehending and receiving messages.

3. The L1 system is utilized in the hypothesis construction responsible for interlanguage development. Again, constraints exist on when transfer takes place.

4. Comprehensible input, including that input which has been make comprehensible with the help of L1 knowledge, serves as a major source of information for hypothesis construction.

5. L2 output, including that output which has been made comprehensible with the help of L1 knowledge, may be used for hypothesis construction.

\section{Conclusion: Where We ARe AND Where We ARe Going}

This paper has examined factors that tend to result in L1 transfer in terms of 7 aspects: Linguistic factors, Psycholinguistic factors, Sociolinguistic factors, Socio-psychological factors, Individual difference, Developmental factors, and Frequency of input.

It should be noticed, however, that factors conductive to L1 transfer are more than what this paper have mentioned, for example, there are studies indicate that gender difference could be a factor resulting in the different extend to which male and female L2 learner would transfer.

More importantly, it should be pointed out that though this paper examined 7 categories of factors separately as if they operate independently; in actuality, however, L1 transfer is a consequence of the co-influence of all of those factors mentioned above, or even more. How these factors interact with each other to shape L2 learners' L1 transfer behavior would be an interesting yet challenging subject of research in the study of language transfer phenomenon.

\section{REFERENCES}


[1] Corder, S. P. (1983). A Role of the Mother Tongue, in Language Transfer in Second Language Learning, Gass, S. M \& Selinker, L (eds.), Philadelphia: John Benjamins Publishing Company.

[2] Ellis, Rod. (1994). Second Language Acquisition, New York: Oxford University Press.

[3] Ellis, Rod. (1994). The Study of Second Language Acquisition, New York: Oxford University Press.

[4] Fuller, Janet M.(1999). Between Three Languages: Composite Structure in Interlanguage, in Applied Linguistics. Oxford University Press.

[5] Larsen-Freeman, D. \& Long, M. (1991). An Introduction to Second Language Acquisition Research, Beijing: Foreign Language Teaching and Research Press 2000.

[6] Kasper, Gabriele \& Faerch, Claus.(1987). Perspectives on Language Transfer, in Applied Linguistics, Vol.8, No.2, summer. Oxford University Press, 72.

[7] Odlin,Terence.(1989). Language Transfer-Cross-linguistic influence in language learning, New York: Cambridge University Press.

[8] Schachter, J.(1988). Second Language Acquisition and Its Relationship to Universal Grammar, in Applied Linguistics, Vol.9, No. 3 Oxford University Press.

[9] Selinker, Larry.(1992). Rediscovering Interlanguage, New York: Longman Inc.

\footnotetext{
Aisong Yi was born in Heze City, China in 1975. She received her M.A. degree in literature from Beihang University, China in 2006.

She is currently a Lecturer in the School of Foreign Languages, Jiangsu University, Zhenjiang, China. Her research interests include English teaching methodology and American literature.
} 